\title{
THICKENING OF THE ULNAR NERVE IN LEPROSY AND ITS TREATMENT
}

\author{
S. N. Chatterji.
}

(Reprinted from "Leprosy in India," Vol. XXIII, No. 1, Jan. 1951)

INTRODUCTION.

Thickening of the ulnar nerve is a well known phenomenon in leprosy. But it is commonly believed that the ulnar nerve is thickened in every case of leprosy. This is not a fact. Patients are sometimes sent to us by medical men with notes of definite diagnosis of leprosy but with an expression of disappointment that they failed to detect any thickening of the ulnar nerve. In those cases there was really no thickening of the ulnar nerve and it was not likely to be thickened as the skin lesions were not in the distribution of this nerve. The reverse is also occasionally seen. That is the nerve is sometimes reported to be thickened when it is not, or it is missed when it is really thickened. This may lead to wrong diagnosis. Another important point is that the detection of thickening of the ulnar nerve has an important bearing in the treatment of leprosy. It is because, if this condition be not detected and properly treated, in due time a patient may be cured otherwise, but he may develop deformities which will remain as a stigma throughout his whole life and, being unable to earn his livelihood, he will remain a burden to the society. Besides that the patient will frequently suffer from blisters and ulcers forming in the affected hand and ultimately there may be loss of digits. This is my apology for introducing this subject.

\section{Sites of Thickening of the Ulnar Ner V.F} and its Relation with SKin Lesions.

This nerve is usually involved when there is anaesthesia or a patch in the ulnar side of the hand. The dorsal branch of the nerve in the hand or near the wrist or the main trunk in the arm may be involved. Sometimes the main trunk is found thickened when there is a patch on the elbow or on the forearm, although these parts are not supplied by the ulnar nerve. The infection is carried to the nerve by lymphatics and this mode of infection is known as the ascending type of infection. Later anaesthesia and deformity may appear in the hand supplied by the nerve. As the main srunk is a mixed nerve and as its involvement causes weakness and deformities of the hand, we shall only refer to the main trunk. 
Mithod of Examination.

The examiner should stand in front of the patient. The patient's elbow should be slightly bent and the examiner should palpate the nerve trunk first in the sulcus nervi ulnaris and then it should be traced upwards. To verify the presence of thïckening, the nerve on the opposite side should be examined and a comparison should be made. It should be remembered that the ulnar nerve is palpable in normal persons. Therefore a nerve should not be considered thickened only because it is palpable. Very rarely the course of the nerve may be found abnormal and it may be found in front of the medical epicondyle. If the examination be not done properly slight thickening of the nerve may be missed or when the nerve is considerably thickened it may be mistaken for a tendon.

PATHOLOGY.

When a nerve is thickened it is swollen. The epineurium is thickened and adherent to the nerve bundles. If the epineurium is incised the affected nerve bundles are found larger in size and pinkish in colour. Nerve fibres are found friable. Yellowish areas indicating caseation may be found in the affected nerve bundles. Thickening of the nerve may be of different degrees. It may be slight, moderate or considerable. Sometimes the thickened nerve is adherent to the surrounding tissues. The nerve may be uniformly thickened or there may be irregular thickening caused by caseation. Sometimes abscesses are found inside the nerve and they may be of different sizes. Big abscesses may sometimes burst through the skin and evacuate spontaneously.

SignS AND SYMPTOMS.

The thickened nerve is usually tender on pressure and even on gentle percussion the patient feels tingling sensation passing down the nerve. However, it should be remembered that tingling sensation may be produced by hard pressure on normal nerves. Sometimes the nerve is acutely inflamed and painful which may keep the patient awake.

When the main trunk is involved certain signs and symptoms are noticed in its distribution sooner or later. The patient feels weakness in the hand. There may be difficulty in side to side movements of the fingers, difficulty in writing and putting on clothing, etc. Later the grip becomes weak and as a result of that he becomes more and more unable to carry on with his work. When the condition is acute there may be pain and burning sensation in the hand and the hand may become hypersensitive to touch.

Gradually wasting of the muscles becomes noticeable. The patient becomes unable to straighten the fingers completely, particularly the little and ring fingers and he is unable to spread the fingers 
in a fan shaped way. In a further advanced condition there is considerable wasting of the muscles supplied by the ulnar nerve, and more bending of the fingers giving rise to typical claw-hand. Ultimately trophic ulcers may appear and may lead to loss of phalanges or loss of digits.

Usually no steps are taken to prevent the development of deformities when there is a chance of such developments and when deformities are already present they are considered permanent lesions and beyond the chance of any improvement with injections. But it has been found out by careful investigation that deformities can usually be prevented from developing, or when they are already there they can be corrected considerably and the suffering of the patient can be mitigated thereby.

\section{TREATMENT.}

The treatment should vary according to the condition of the nerve and of the muscles supplied by the nerve, presence of pain, acute inflamation or abscess formation. It should also be considered whether the nerve pain is due to general lepra reaction. When there is lepra reaction the patient should be treated for that condition with calcium and antimony preparations. With the subsidence of reaction, skin lesions subside and thickening of the nerve goes down, at least to some extent, and the patient is relieved of the pain.

When there is no lepra reaction and when the nerve is slightly thickened and tender, injections of hydnocarpus oil in small doses subcutaneously by the side of the nerve and also in the affected hand are found useful.* Usually this is not done and injections are given only in skin lesions and not by the side of the nerve and when active lesions subside and when there is only slight hypopigmentation, anaesthesia and deformity of the hand injections are either stopped or given only intramuscularly in the buttocks. The result is that gradually there is more anaesthesia, wasting and deformity of the part and frequent blisters and ulcers form, causing endless trouble to the patient. Therefore along with the injections in other parts, injections should also be given in the affected hand and also by the side of the nerve from time to time. The treatment should be continued even when active lesions subside. This should be done not with a view to have any specific action of the hydnocarpus oil but to stimulate the muscles of the hand and reduce the thickening of the nerve.

The patient should be advised to exercise the hand, if possible with a pair of spring dumb-bells and also massage the hand with

"(This possibly acts as a form of counter-irritation, and must be applied with great care.-EDitor) 
some oil daily for I hour. Kneading of the muscles, rubbing of the fore-arm and hand from above downwards and pulling of the fingers are necessary.* The hand should be protected from too much heat and cold. Therefore the patient should be advised not to touch anything hot and avoid going near a fire and also use woollen gloves in cold weather. Usually these measures are a valuable aid to the prevention of deformities and ulcerations. Even cases showing slight signs of deformities may benefit by this treatment.

When deformities are more advanced or when there is nerve abscess or acute nerve pain, not relieved by other methods, decapsulation of the nerve should be done without delay to prevent further deformity. Subsequently routine treatment, massage and exercise etc. as described before should be followed to correct the residual deformity and weakness etc. not being corrected by operation alone. Splinting of the hand is alsc another useful measure. In many cases encouraging results have been obtained by following this combined treatment. There was complete relief of pain in the majority of cases. In some cases deformities were entirely corrected and there was complete return of strength. In other cases there was partial improvement, and further deterioration, appearance of trophic ulcers and loss of digits were prevented. In most of the cases there was some return of sensation and therefore the incidence of accidental burn and blister formation became less and less. In a small number of lepromatous cases who suffered from repeated reaction this method of treatment gave only temporary relief. But subsequent reaction made their condition worse.

Decapsulation or removal of the sheath of the nerve can be done under local anaesthesia. The nerve is situated behind the intramuscular septum which should be incised. Then the epineurium should be incised and dissected off carefully all round the thickened portion ( 2 to 4 inches) of the nerve in the arm. The rationale of this operation is that the nerve sheath becomes very dense and unyielding and causes pressure on the nerve fibres with the resulting severe pain and later there is degeneration and atrophy of the nerve fibres and of the muscles supplied by them. Subsequently there may be loss of digits which makes the patient completely crippled. Removal of the sheath of thickened nerve relieves tension inside the nerve bundles and thus prevents the unhappy sequence of events. But we should not depend upon this operation alone. This is only a part of the treatment. It should be combined with routine treatment of leprosy, massage and exercise of the affected hand.

"The method of massage and splinting is detailed in Dr. Brand's article (p.51). 


\section{ILLUSTRATIVE CASES}

(i) P.K., Case No. 7521 .

Early neural case.

Duration $6 \frac{1}{2}$ years

\section{Before treatment}

24.9.43.

Lesion on the rt. little finger. Rt. ulnar

+++ (caseous). Pain in rt. ulnar and

rt. hand. Weakness in rt. hand. Difficulty

in writing.

12.1.45.

Lesion faded completely. Muscular strength considerably restored. Slight return of sensation. Thickening of the ulnar nerve less than before.

\section{After treatment}

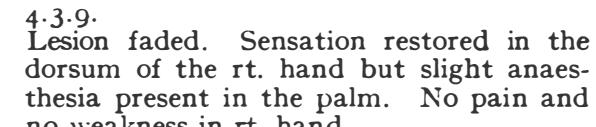
no weakness in $\mathrm{rt}$. hand.

(ii) R.D. Case No. 8658 .

classification doubtful.

$\left(\mathrm{NS}_{2} \mathrm{a}_{0}+\right.$ ? $\left.\mathrm{L}\right)$

Before treatment. Fig. 1 (a)

$20.7 \cdot 48$

Rt. ulnar ++

Rt. little, middle and ring fingers are bent.

Difficulty in writing. Veakness in rt. hand.

Difficulty to raise heavy things. Feels shy

After treatment Fig. 1 (b)

22.2.49

Out of four fingers two fingers are now bent.

(Rt. little and ring fingers bent.)
24. I. 50.

Very slight bending of $\mathrm{rt}$. little and ring fingers and therefore he can now go out without raising suspicion and without feeling shy. No difficulty in writing. Hand writing has improved. He can grip properly and raise heavy things. Lesion faded considerably and there is some return of sensation.

(iii) G.R., Case No. 827 I

Early neural case.

Duration 4 years.

Machine assistant.

Before treatment.

I I.6.46.

Rt. ulnar ++

Pain in rt. hand. Difficulty in writing. Difficulty in doing manual

work. He was selected for decapsulation failing improvement with

7.I.5O.

After treatment.

Lesion faded, Rt. ulnar less thick. Pain subsided. Return of strength in $\mathrm{rt}$. hand. Some return of sensation. Therefore decapsulation was not done. 
(iv) M.M., Case No. 8526 .

Early neural case.

Duration $2 \frac{1}{2}$ years.

Before treatment.

I 8.10 .47 .

Lt. ulnar ++

Pain in left ulnar and in left hand. Weakness in left hand and

therefore difficulty in manual work, difficulty in putting on clothing

and in ablutions after defaecation.

(v) R.P.S. Case No. 869I.

Neural case, moderately advanced.

Duration 2 years. Draftsman.

Before treatment.

21.9 .48 .

Left ulnar +++

Pain in left ulnar. Pain and burning sensation in left hand Weak

ness in left hand and therefore could not grip with the left hand or

straighten the fingers properly. Therefore he felt it difficult to do the work of a draftsman.

Present condition after treatment.

\section{After treatment.}

Left ulnar still thick. Pain relieved. Normal strength in left hand No difficulty in doing any work.

(vi) S.M. case No. 8990-Fig. 2 (a)

Neural case.

Duration 5 years 8 months.

\section{Before treatment.}

23. I I.49.

Slightly red, thick and anaesthetic lesion covering the left hand,

forearm and arm. Left little, ring, middle and index fingers are

bent. Left ulnar and left superficial radial nerves thickened.

I0. I.50.

After treatment.

Lesions subsided Pain in the nerve and left hand relieved. Increased strength in left hand. $\mathrm{He}$ can now grip with the left hand and straighten the fingers properly. Therefore there is not much difficulty in doing his work.

\section{After treatment. Fig. 2 (b)}

$4 \cdot 7 \cdot 50$.

Lesion subsided considerably. It is now hypopigmented and flat. Sensation restored in some places. Excepting the left index finger which is still slightly bent other fingers have become straight. Increased strength in left hand. Left ulnar and left superficial nerves less thick.

(i) Sk. H., Case No. 7403 . Duration $6 \frac{1}{2}$ years.

Before treatment.

$7 \cdot 5 \cdot 43$.

Rt. ulnar ++

(caseous)

Pain in rt. ulnar and rt. little finger. Rt.

little finger bent. Loss of strength in rt.

hand. Difficulty in writing. Could not

Weakness and deformities corrected by decapsulation combined with other treatment.

grip.

$25 \cdot 5 \cdot 43$

Decapsulation done.

After treatment.

Nerve less thick.

More strength in $\mathrm{rt}$.

hand. Rt. little tin-

ger less bent.
30. I. 50

No pain in rt. ulnar and rt. little finger. Deformity of the right little finger cor rected considerably. Return of strength in rt hand. No difficulty in writing and the grip is normal. Slight wasting of muscles present but further deformity prevented. 
(ii) S.D., Case No. 5088

Motor Car Driver.

Early neural case.

Duration 14 years.

Before treatment.

r9.8.35.

Left ulnar ++

Weakness in left hand.

driving motor car.

22.8 .35

Injections started

28. I.39.

Slight deformity of

left hand noticed.

\section{After treatment.}

28.r.39.
Decapsulation done.

30.1 .50

Lesion faded. Considerable return of sensation. Almost normal strength in left hand. Slight wasting present. No difficulty in driving motor car.

(iii) B., Case No. 7950.

Early neural case.

Duration 5 years.

Before treatment. Fig. 3 (a)

$8 \cdot 5 \cdot 45$.

Rt. ulnar +++

I7:5.45.

Injection

started.

$25 \cdot 5 \cdot 45$.

Decapsulation

done.

little, ring and middle fingers bent.

Difficulty in putting on clothings.

Difficulty in doing manual work. Could

not take food with his rt. hand. Used

to feed himself wiţh his left hand.

\begin{abstract}
I8. I. 46 .
Lesion faded.

Rt. middle and

ring finger are

now straight.

Slight bending

of the rt. little

finger.

No pain.
\end{abstract}

(c) Improvement noticed soon after decapsulation. in $\mathrm{rt}$. hand.
I 3.6.47. More strength

$24 \cdot 9 \cdot 49$.

After treatment. Fig. 3 (b)

Further improvement. All the three fingers have become straight. Therefore correction is complete. No diffi. culty in doing work.

(i) G.R.M., Case No. 8998.

Moderately advanced neural case.

Duration 8 months.

A girl of 15 years.

Before treatment.

25. I I.49.

Rt. ulnar +++

Right little, ring, middle and index fingers bent.

Could not grip or do any work or feed herself. Could

not even raise a glass of water. Could not write.

$\mathrm{Pain}$ in rt. hand and rt. ulnar.

\section{After treatment.}

I3. I 2.49 .

Decapsulation done.
I.2.50.

Deformity in rt. hand less. Fingers not so bent as before. Some return of strength in rt. hand. She can now grip and do some work, although the grip is not so strong. No pain and tingling sensation in $\mathrm{rt}$ hand and in rt. ulnar. She can now write, feed herself and raise buckets full of water with her right hand. 
R. D. Case No. 8658. Figure I (a).

R. D. Case No, 8658. Figure 1 (b)

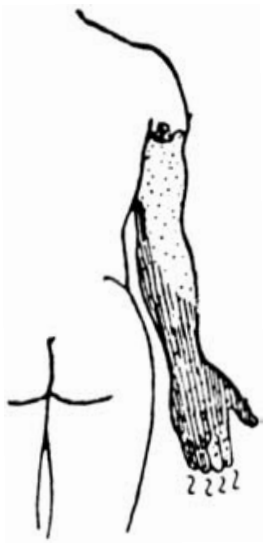

Refore Treatment.

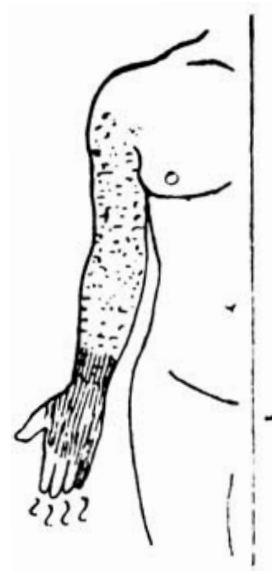

$N, B .-\mathrm{S}$
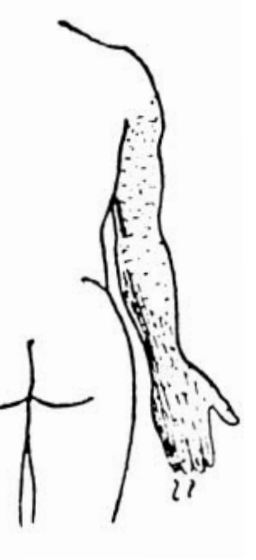

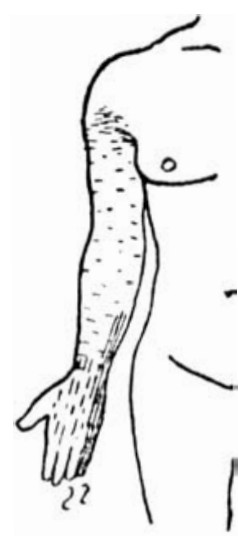

After Treatment.

(1) Anaesthesia

(4) Erytbema and thickening

(2) Bent fingers $\delta \delta$

(3) Hypopigmentation

(5) Thickened nerve

B, Case No. 7950.

Figure 3 (a).

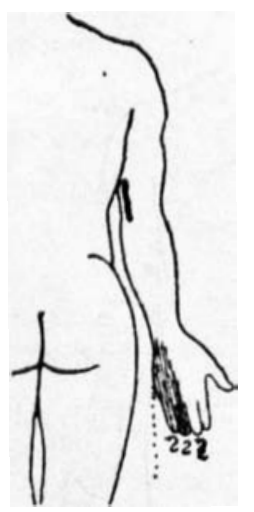

Before Treatment. N. B.-Symbols used for different sigas :-

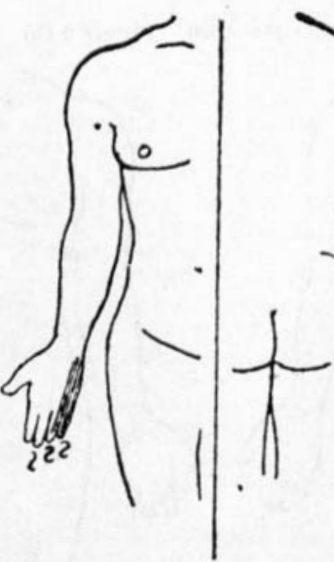

Figure No. 3 (b)

(1) Anaesinesia \|\|\|\| (2) Bent fagers $\delta \delta$

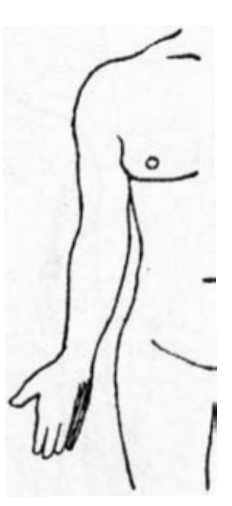

(4) Erytbema and tbickering

itit

(3) Hypopigmentation

(5) Tbickened nerve 
S. M. Case No 8990. Figure 2 (a)
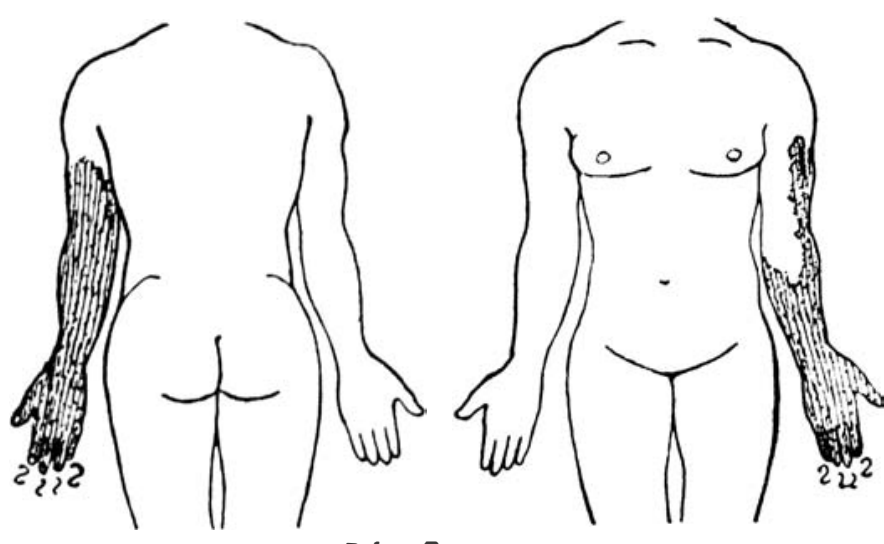

Before Treatment.

N. B.-Symbols used for different signs :-

(1) Anaesthesia

(2) Bent fingers $\delta \delta$

(3) Hypopigmentatton

(4) Erytbema and tbickening

S. M. Case No. 8990 . Figure 2 (b)
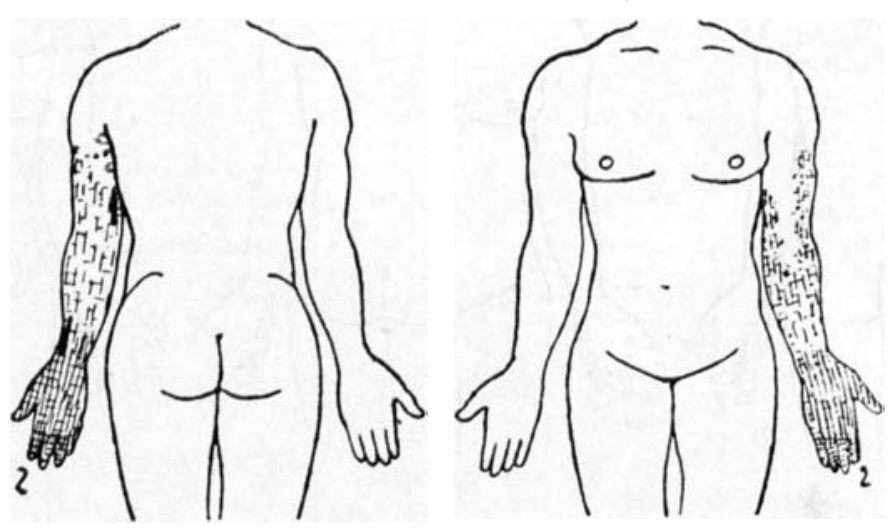

After Treatment.

N. B.-Symbols used for different signs :-

(1) Anaesthesia \|\|\|\| (2) Bent fogers $\delta \delta$

(4) Ergibema and thickening is

(5) Thickened nerve 
Conclussions.

Deformity of the hand is a serious complication of leprosy as it may make the patient an invalid and unable to earn his livelihood. Therefore it should be the endeavour of all leprosy workers to prevent this complication or try to correct it as much as possible by all means. As the claw hand is caused by involvement of ulnar nerve the pathology of the process should be clearly understood to administer proper treatment. In this paper we have emphasised the benefit which may be derived by giving injections of hydnocarpus oil, massage, exercise and decapsulation etc. Other methods of treatment like diathermy application, stimulation of muscles by galvano-faradic current, massage by specialists or special baths, have not been discussed here because, although useful, only a small number of patients in big institutions can have the benefit of these treatments. The methods advocated in this article are simpler, do not require any special apparatus or a specialist, and can be easily carried on in any outdoor clinic. If properly carried on with patience and perseverance for a long time patients will benefit considerably as will be evident from the cases illustrated here. 